\title{
Regulatory effect of microRNA-223-3p on breast cancer cell processes via the Hippo/Yap signaling pathway
}

\author{
TONGHUA DU, DAN WANG, XIAOYU WAN, JINGWEI XU, QI XIAO and BIN LIU \\ Department of Breast Surgery, The Second Hospital of Jilin University, Changchun, Jilin 130041, P.R. China
}

Received February 7, 2020; Accepted December 17, 2020

DOI: 10.3892/ol.2021.12777

\begin{abstract}
According to the 2018 global cancer statistics, the incidence and mortality rates of breast cancer are increasing gradually, which seriously threatens the health of women. MicroRNA-223-3p (miR-223-3p) can promote the proliferation and invasion of breast cancer cells. Hippo/Yes-related protein (Yap) signaling pathway activation has been found in a variety of tumors. The present study aimed to investigate the potential mechanism of miR-223-3p in breast cancer. The Cell Counting Kit- 8 assay was used to detect cell viability and flow cytometry was used to detect apoptosis. The abilities of cell migration and invasion were detected using scratch and Transwell assays, as well as reverse transcription-quantitative PCR and western blotting to detect gene and protein expression, respectively. The current results demonstrated that miR-223-3p transcription levels were increased in breast cancer cells, and inhibition of miR-223-3p gene expression decreased cell proliferation, migration and invasion. Additionally, inhibition of miR-223-3p expression inhibited epithelial-mesenchymal transition (EMT) in breast cancer cells. miR-223-3p promoted cell proliferation, migration, invasion and EMT, and the western blotting results demonstrated that miR-223-3p inhibition increased the phosphorylation of Yap1 and the protein expression levels of large tumor suppressor kinase 1. In conclusion, results from the present results suggested that miR-223-3p may promote cell proliferation, migration, invasion and EMT through the Hippo/Yap signaling pathway. Therefore, miR-223-3p may be a potential biomarker for breast cancer.
\end{abstract}

\section{Introduction}

According to the 2018 global cancer statistics, the incidence rate of breast cancer in women accounts for $11.6 \%$ of the total

Correspondence to: Professor Bin Liu, Department of Breast Surgery, The Second Hospital of Jilin University, 218 Ziqiang Street, Nanguan, Changchun, Jilin 130041, P.R. China

E-mail: liubin19810713@protonmail.com

Key words: breast cancer, epithelial-mesenchymal transition, microRNA-223-3p, Hippo/Yes-related protein pathway cancer cases (1). Breast cancer is the most common cancer among women, and is also the leading cause of cancer-associated deaths (1). In 2014, the incidence rate of breast cancer in China was $18.65 \%$, and it is growing at a rate of $3 \%$ per year (2), which has become one of the main threats to the health of women; it is a great burden on society and requires further research. There are numerous treatments for breast cancer, including targeted molecular therapy, chemotherapy, radiotherapy and surgery (3). Although considerable progress has been achieved in the medical treatment of breast cancer, there is no radically effective treatment for patients with breast cancer, and the prognosis of clinical treatment remains extremely poor. Additionally, breast cancer, especially triple-negative breast cancer, is prone to recurrence and metastasis (4). Therefore, in-depth study of breast cancer and the identification of novel biomarkers or active therapeutic targets are particularly important research directions.

MicroRNA (miRNA/miR)-223-3p is located in the q12 locus of the $\mathrm{X}$ chromosome (5). miR-223-3p is highly conserved and has potential roles in major physiological changes. For example, miR-223-3p acts as an oncogene in certain cancer types, such as T-cell acute lymphoblastic leukemia (6), gastric cancer (7) and prostate cancer (8), whereas in acute myeloid leukemia (9), cervical cancer (10) and non-small cell lung cancer (11) it functions as a tumor suppressor. Currently, the role of miR-223-3p in tumorigenesis has not been fully determined. Therefore, it is important to understand the role of miR-223-3p in tumorigenesis and the development of disease. It has been revealed that miR-223-3p has the potential to promote the proliferation and invasion of cancer cells. For instance, the proliferation and invasion of breast cancer cells are enhanced following transfer of miR-223-3p into cells (12). However, to the best of our knowledge, the mechanism of miR-223-3p in breast cancer has not been previously described.

Epithelial-mesenchymal transition (EMT) is a reversible cellular process that temporarily puts epithelial cells in a quasi-mesenchymal state (13). EMT is associated with wound healing, tissue regeneration and organ fibrosis, and is involved in metastasis and cancer progression (14). In addition, EMT activation in tumor cells affects the development of various types of malignancies, such as breast and pancreatic cancer $(15,16)$. During tumor development, this pleiotropic process induces, in a single cancer cell, multiple features associated with advanced malignancies $(17,18)$. Metastatic breast cancer is largely incurable (19). Therefore, increased understanding of 
EMT regulation in breast cancer may enable the development of novel targeted treatment strategies. However, to the best of our knowledge, the regulatory mechanisms of miR-223-3p and EMT in breast cancer have not been studied.

The Hippo signaling pathway was first discovered in Drosophila. It can inhibit cell proliferation and regulate apoptosis to limit the size of tissue morphological growth (20). In addition to regulating stem/progenitor cell expansion, inhibiting cell proliferation, stimulating apoptosis and controlling organ size, the Hippo signaling pathway also serves a crucial role in the proliferation of tumor cell proliferation (21). Yes-related protein (Yap) is a key effector in the Hippo signaling pathway. It has been reported that the Hippo signaling pathway is inactivated by inhibition of Yap activity, and increased Yap activity can inhibit the expression of tumor suppressor genes in tumor cells (22). EMT and the Hippo signaling pathway serve a regulatory role in the proliferation and growth of tumor cells (23). However, to the best of our knowledge, the role of the Hippo signaling pathway in breast cancer has not been previously determined. Therefore, the roles of EMT and the Hippo signaling pathway are novel research targets in breast cancer.

The present study aimed to investigate the effects of miR-223-3p on the proliferation, migration and invasion of breast cancer cells via the Hippo/Yap signaling pathway, and to further evaluate the underlying mechanism of miR-223-3p in breast cancer cells to provide a research basis for the clinical treatment of breast cancer.

\section{Materials and methods}

Cell culture. Human breast cancer MDA-MB-231 and MCF-7 cells (both purchased from Peking Union Medical College) were cultured in DMEM (Gibco; Thermo Fisher Scientific, Inc.) containing 10\% FBS (Invitrogen; Thermo Fisher Scientific, Inc.), $100 \mathrm{U} / \mathrm{ml}$ penicillin and $100 \mu \mathrm{g} / \mathrm{ml}$ streptomycin. Human normal mammary epithelial cells (MCF-10A, purchased from Peking Union Medical College) cells were cultured in growth medium consisting of DMEM/F-12 (1:1; Gibco; Thermo Fisher Scientific, Inc.) supplemented with 10\% FBS, $100 \mathrm{U} / \mathrm{ml}$ penicillin and $100 \mathrm{U} / \mathrm{ml}$ streptomycin. Cells were incubated at $37^{\circ} \mathrm{C}$ in a $5 \% \mathrm{CO}_{2}$ atmosphere. Cells were cultured at saturated humidity and $37^{\circ} \mathrm{C}$, changing culture medium every 2 days. When cells grew to $90 \%$, they were trypsinized (25\%) and then centrifuged for $10 \mathrm{~min}$ at $4^{\circ} \mathrm{C}$ and $18,750 \mathrm{x}$ g before resuspension. The suspension was added to a new petri dish.

Total RNA extraction and reverse transcription-quantitative $P C R(R T-q P C R)$. Total RNA was extracted from cells using TRIzol $^{\circledR}$ reagent (Invitrogen; Thermo Fisher Scientific, Inc.) or the Ultrapure RNA kit (CoWin Biosciences) according to the manufacturer's protocols. RNA $(0.5 \mu \mathrm{g})$ was subjected to reverse transcription using ReverTra Ace qPCR RT Master mix with gDNA Remover (Toyobo Life Science). After adding oligo(dT) into RNA, it was heated at $70^{\circ} \mathrm{C}$ for $10 \mathrm{~min}$ and put in an ice bath for $1 \mathrm{~min}$; $\mathrm{m}-\mathrm{mlv}$, dNTP and rnasin were then added, mixed well and centrifuged, then stored at $42^{\circ} \mathrm{C}$ for $40-90 \mathrm{~min}$, $95^{\circ} \mathrm{C}$ for $10 \mathrm{~min}$ and finally at $4^{\circ} \mathrm{C}$. qPCR was performed using a Bio-Rad IQ5 instrument (Bio-Rad Laboratories, Inc.) with TransStart ${ }^{\circledR}$ Top Green qPCR SuperMix (Beijing Transgen
Biotech Co., Ltd.). The reactions were performed under the following conditions as suggested by the manufacturer: $94^{\circ} \mathrm{C}$ for $30 \mathrm{sec}$, followed by 40 cycles of $94^{\circ} \mathrm{C}$ for $5 \mathrm{sec}$ and $60^{\circ} \mathrm{C}$ for $30 \mathrm{sec}$, followed by a dissociation protocol. The following primer sequences were used: miR-223-3p forward, 5'-TAA AGCAACCGAGCACTGAGA-3' and reverse, 5'-ACGGTA GAGGTCCTTTCCTTTG-3'; and 18S rRNA forward, 5'-AGG CGCGCAAATTACCCAATCC-3' and reverse, 5'-GCCCTC CAATTGTTCCTCGTTAAG-3'. The relative expression was normalized to $18 \mathrm{~S}$ rRNA expression (24), used as a loading control. Relative gene expression was analyzed using the $2^{-\Delta \Delta C a}$ method (25).

Cell transfection assay. MCF-7 cells were cultured in a 6-well plate at $37^{\circ} \mathrm{C}$ for $24 \mathrm{~h}$. Transfection was performed when the cell density grew to $\sim 70 \%$. Subsequently, cells were transfected with miRVana miRNA inhibitors of miR-223-3p (miR-223-3p inhibitor; $50 \mathrm{nM}$; cat. no. 4464084; Thermo Fisher Scientific, Inc.) and negative control (NC; $50 \mathrm{nM}$; cat. no. R10034; Guangzhou RiboBio Co., Ltd.) using Lipofectamine ${ }^{\circledR} 2000$ (Thermo Fisher Scientific, Inc.), according to the manufacturer's protocol, for $6 \mathrm{~h}$ at $37^{\circ} \mathrm{C}$. Subsequently, the medium was changed to DMEM containing $10 \%$ serum and incubated at $37^{\circ} \mathrm{C}$ for $12 \mathrm{~h}$ before subsequent experiments (26).

Cell proliferation assay. A Cell Counting Kit 8 (CCK8; Beyotime Institute of Biotechnology) assay was used to detect the proliferation of mammary cancer cells. Briefly, at a cell growth density of $\sim 70 \%$, control (untransfected cells), NC- and miR-223-3p inhibitor-transfected MCF-7 cells were cultured in 96 -well cell culture plates. After incubation at $37^{\circ} \mathrm{C}$ for 12 , 24, 48 and $72 \mathrm{~h}, 10 \mu \mathrm{l}$ CCK8 reagent was added to each well. The cells were placed in a cell incubator at $37^{\circ} \mathrm{C}$ for $0.5-1.5 \mathrm{~h}$. Subsequently, the optical density value of each well was determined on a microplate reader at $450 \mathrm{~nm}$.

Apoptosis assay. The cells were cultured in 6-well plates and divided into three groups: Control group (untransfected MCF-7 cells), NC group and miR-223-3p inhibitor MCF-7 group. After transfection, $1 \times 10^{6}$ cells were collected, washed in $1 \mathrm{ml}$ $1 \mathrm{X}$ binding buffer and centrifuged at $4^{\circ} \mathrm{C}$ and $18,750 \mathrm{x}$ g for $10 \mathrm{~min}$. Subsequently, $1 \mathrm{ml} 1 \mathrm{X}$ binding buffer was added to the collected cells, and then $10 \mu \mathrm{l}$ Annexin V-FITC (Sungene Biotech Co., Ltd.) reagent was added to each group of collected cells. After incubation in the dark at $37^{\circ} \mathrm{C}$ for $15 \mathrm{~min}, 5 \mu \mathrm{l} \mathrm{PI}$ (Sungene Biotech Co., Ltd.) solution was added for $10 \mathrm{~min}$ at room temperature and apoptosis was analyzed by flow cytometry (CytoNova; CapitalBio Technology, Inc.). Results were analyzed using the Kaluza software (v2.1.1; Beckman Coulter, Inc.). The apoptotic rate was calculated as the percentage of early and late apoptotic cells.

Cell migration assay. Cell migration was measured using a wound healing assay. A total of $5 \times 10^{4}$ cells were cultured in each well of a 24-well plate and cultured overnight in the incubator at $37^{\circ} \mathrm{C}$ to enable the cells to adhere to the bottom of the plate. Once the cells grew to $\sim 80 \%$ confluence, a straight-line scratch was made with a $200-\mu 1$ pipette tip, followed by washing with PBS and the medium was replaced with FBS-free medium. Subsequently, the scratch area was imaged 

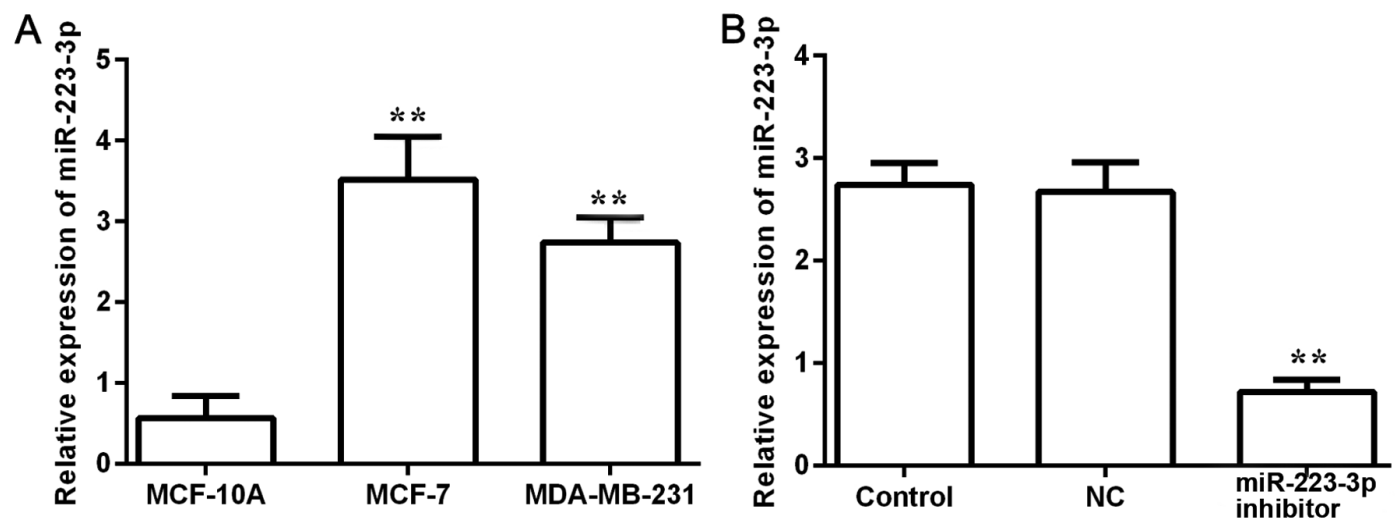

Figure 1. miR-223-3p expression in breast cancer cells. (A) Expression levels of mir-223-3p in MCF-10A, MCF-7 and MDA-MB-231 cells was detected by RT-qPCR. ${ }^{* *}$ P $<0.01$ vs. MCF-10A cells. (B) Expression levels of miR-223-3p in MCF-7 transfected with miR-223-3p inhibitor was detected by RT-qPCR. Data are presented as the mean $\pm \mathrm{SD}$ of three experiments. ${ }^{* *} \mathrm{P}<0.01$ vs. Control. miR, microRNA; NC, negative control; RT-qPCR, reverse transcription-quantitative PCR; siRNA, small interfering RNA.

using an optical light microscope (Olympus CKX53; Olympus Corporation; magnification, x100) to record the results at $0 \mathrm{~h}$. The cells were then placed in a cell incubator. After $24 \mathrm{~h}$ at $37^{\circ} \mathrm{C}$, cell migration area size was analyzed with ImageJ software (v1.8.0; National Institutes of Health), comparing the findings with the previous time point. The experiments were repeated three times.

Cell invasion assay. The invasive ability of cells was examined using a Transwell (Corning, Inc.) assay. The upper surface of the bottom membrane of the Transwell chamber was coated with $50 \mathrm{mg} / \mathrm{l}$ Matrigel $(1: 8)$ for $30 \mathrm{~min}$ at $37^{\circ} \mathrm{C}$ and air dried at $4^{\circ} \mathrm{C}$. The residual liquid in the culture plate was aspirated and $50 \mu \mathrm{l}$ serum-free culture medium containing $10 \mathrm{~g} / \mathrm{l} \mathrm{BSA}$ (Thermo Fisher Scientific, Inc.) was added into each well at $37^{\circ} \mathrm{C}$ for $30 \mathrm{~min}$. The chamber was placed in the culture plate, and $300 \mu \mathrm{l}$ pre-warmed serum-free medium was added to the upper chamber, which was left at room temperature for 15-30 min to rehydrate the matrix gel and absorb the remaining culture medium. Cells were starved in serum-free medium for 12-24 h before preparing the cell suspension. Cells were added to 24-well culture plates and Transwell chambers were placed in them. Moreover, $500 \mu 1$ medium containing FBS was added to the lower chamber of the orifice plate. The cells were cultured at $37^{\circ} \mathrm{C}$ for $24 \mathrm{~h}$. The cells invading the chamber were stained with $0.1 \%$ crystal violet for $1 \mathrm{~h}$ at room temperature, and 3-5 fields of view were randomly selected for imaging. Stained cells were counted under an optical light microscope (Olympus CKX53; Olympus Corporation; magnification, $\mathrm{x} 100)$.

Western blotting. Proteins were extracted from treated cells using lysis buffer (Beyotime Institute of Biotechnology) and quantified using a bicinchoninic acid protein assay kit (Beyotime Institute of Biotechnology). A total of $10 \mu \mathrm{g}$ protein/lane was separated by $10 \%$ SDS-PAGE and then transferred to PVDF membranes. The membranes were blocked with $5 \%$ skimmed milk at room temperature for $60 \mathrm{~min}$ and incubated with primary antibodies at $4^{\circ} \mathrm{C}$ overnight. Rabbit monoclonal primary antibodies against GAPDH (cat. no. ab181602), macrophage stimulating 1 (MST1; cat. no. ab79199), large tumor suppressor kinase 1 (LATS1; cat. no. ab70561), phosphorylated-Yap1 (cat. no. ab76252), Yap1 (cat. no. ab52771) and MMP14 (cat. no. ab51074), and the anti-rabbit immunoglobulin G secondary antibody (cat. no. ab7090) were obtained from Abcam. MMP9 (cat. no. 10375-2-AP), MMP2 (cat. no. 10373-2-AP), CDK2 (cat. no. 10122-1-AP), Cyclin E1 (cat. no. 11554-1-AP), cyclin-dependent kinase inhibitor 1 (p21; cat. no. 10355-1-AP), E-cadherin (cat. no. 20874-1-AP), $\mathrm{N}$-cadherin (cat. no. 22018-1-AP) and vimentin (cat. no. 10366-1-AP) antibodies were obtained from ProteinTech Group, Inc. All antibodies were diluted as recommended in the specifications (dilution ratio, 1:1,000). The blots were then incubated with HRP-conjugated secondary antibody $(1: 2,000$; cat. no. 7074; Cell Signaling Technology, Inc.) at room temperature for $1 \mathrm{~h}$. After extensive washing in TBST, protein bands were revealed with Super Signal West Femto Maximum Sensitivity Substrate (Thermo Fisher Scientific, Inc.) and visualized with Image Quant LAS 500 (Cytiva).

Statistical analysis. All results were obtained from $\geq 3$ independent experiments and data are presented as the mean \pm SD. Data were analyzed using SPSS software (version 20.0; IBM Corp.). GraphPad Prism software (version 6.0; GraphPad Software, Inc.) was used to generate figures. One-way ANOVA was used to analyze the significance of differences among different groups. Comparisons between multiple groups were performed using the Scheffe post-hoc test. $\mathrm{P}<0.05$ was considered to indicate a statistically significant difference.

\section{Results}

miR-223-3p expression levels in different breast cancer cells. miR-223-3p expression levels were detected in MCF-10A normal breast cells and in two different breast cancer cell lines, MCF-7 and MDA-MB-231 (Fig. 1A). The expression levels of miR-223-3p in breast cancer cells were significantly higher compared with expression in normal cells. As the highest miR-223-3p expression level was observed in MCF-7 cells, these cells were selected for subsequent experiments. After miR-223-3p inhibitor was transfected into MCF-7 cells, miR-223-3p expression was detected (Fig. 1B). The 

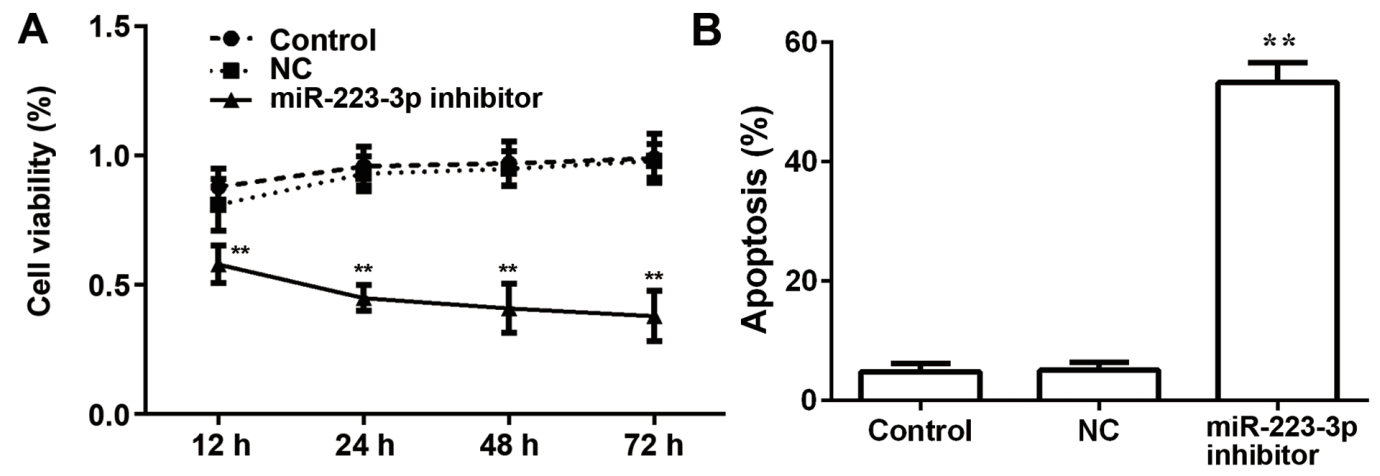

C
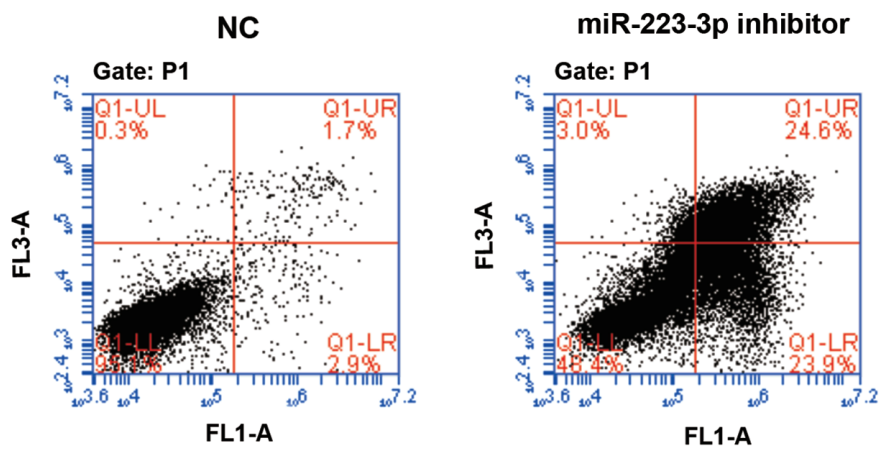

Figure 2. Effects of miR-223-3p inhibition on the proliferation and apoptosis of MCF-7 cells. (A) After transfection, the proliferation curve of MG-63 cells was determined using a Cell Counting Kit 8 assay. Optical density values at $450 \mathrm{~nm}$ were detected at 12, 24, 48 and $72 \mathrm{~h}$. (B) Apoptosis data statistics. (C) Annexin V-FITC/PI staining was used to detect the apoptosis of cells after $24 \mathrm{~h}$. Data are presented as the mean \pm SD of three experiments. ${ }^{* *} \mathrm{P}<0.01$ vs. Control. miR, microRNA; NC, negative control; siRNA, small interfering RNA.

results demonstrated that miR-223-3p inhibitor could inhibit miR-223-3p expression.

Downregulation of miR-223-3p inhibits the proliferation of breast cancer cells in vitro. To examine the role of miR-223-3p on the proliferation of MCF-7 cells, miR-223-3p inhibitor was transfected into MCF-7 cells and results were compared with the control group. Subsequently, cell viability was detected using a CCK8 assay at different time points, and a growth curve was generated based on the results. As presented in Fig. 2A, there was no significant difference in the proliferation rate between the MCF-7 cell control group and the NC group, whereas the cell proliferation rate of the miR-223-3p inhibitor group was significantly lower compared with that of the control group. By detecting the apoptosis rate, it was found that inhibiting miR-223-3p expression significantly increased apoptosis (Fig. 2B and C).

Subsequently, the expression levels of cell cycle-related proteins CDK-2, cyclin-related proteins Cyclin E1 and p21 were examined to further evaluate the effect of miR-223-3p on cell proliferation. Compared with the control group, the protein expression levels of CDK-2 and Cyclin E1 were downregulated, whereas the expression of p21 was upregulated in the miR-223-3p inhibitor group (Fig. 3). These results suggested that inhibiting miR-223-3p expression could inhibit the proliferation of breast cancer cells.

miR-223-3p inhibition suppresses the migration and invasion of breast cancer cells. The effects of miR-223-3p on the migration and invasion of MCF-7 cells were investigated. Results from the wound healing assay demonstrated that loss of miR-223-3p expression significantly inhibited the migratory ability of MCF-7 cells (Fig. 4A and C). Similarly, Transwell analysis indicated that the invasive capacity of cells transfected with miR-223-3p inhibitor was significantly declined compared with that of the control group (Fig. 4B and D). Furthermore, in cells transfected with miR-223-3p inhibitor, the protein expression levels of metastasis-associated proteins, including MMP9, MMP2 and MMP14, were significantly decreased compared with the control group expression levels (Fig. 5). These data suggested that inhibiting miR-223-3p expression may decrease migration and invasion in MCF-7 cells.

Inhibition of mir-223-3p decreases EMT in breast cancer cells. The expression levels of EMT-associated proteins were examined to determine the effects of miR-223-3p on EMT in breast cancer cells. The results suggested that inhibiting the expression of miR-223-3p increased the protein expression of E-cadherin, and decreased N-cadherin and vimentin expression levels (Fig. 6). Thus, these data indicated that inhibition of miR-223-3p may reverse EMT in breast cancer cells.

miR-223-3p and the Hippo/Yap signaling pathway. The western blotting results demonstrated that the expression levels of phosphorylated Yap1 were increased in MCF-7 cells transfected with miR-223-3p inhibitor compared with those in control cells (Fig. 7A and D). Moreover, no significant difference was found between the protein expression levels of MST1 in cells transfected with miR-223-3p inhibitor and control cells (Fig. 7A and B). It was identified that LATS1 expression was significantly upregulated in miR-223-3p inhibitor-transfected cells (Fig. 7A and C). These results suggested that miR-223-3p may activate the Hippo/Yap signaling pathway. 

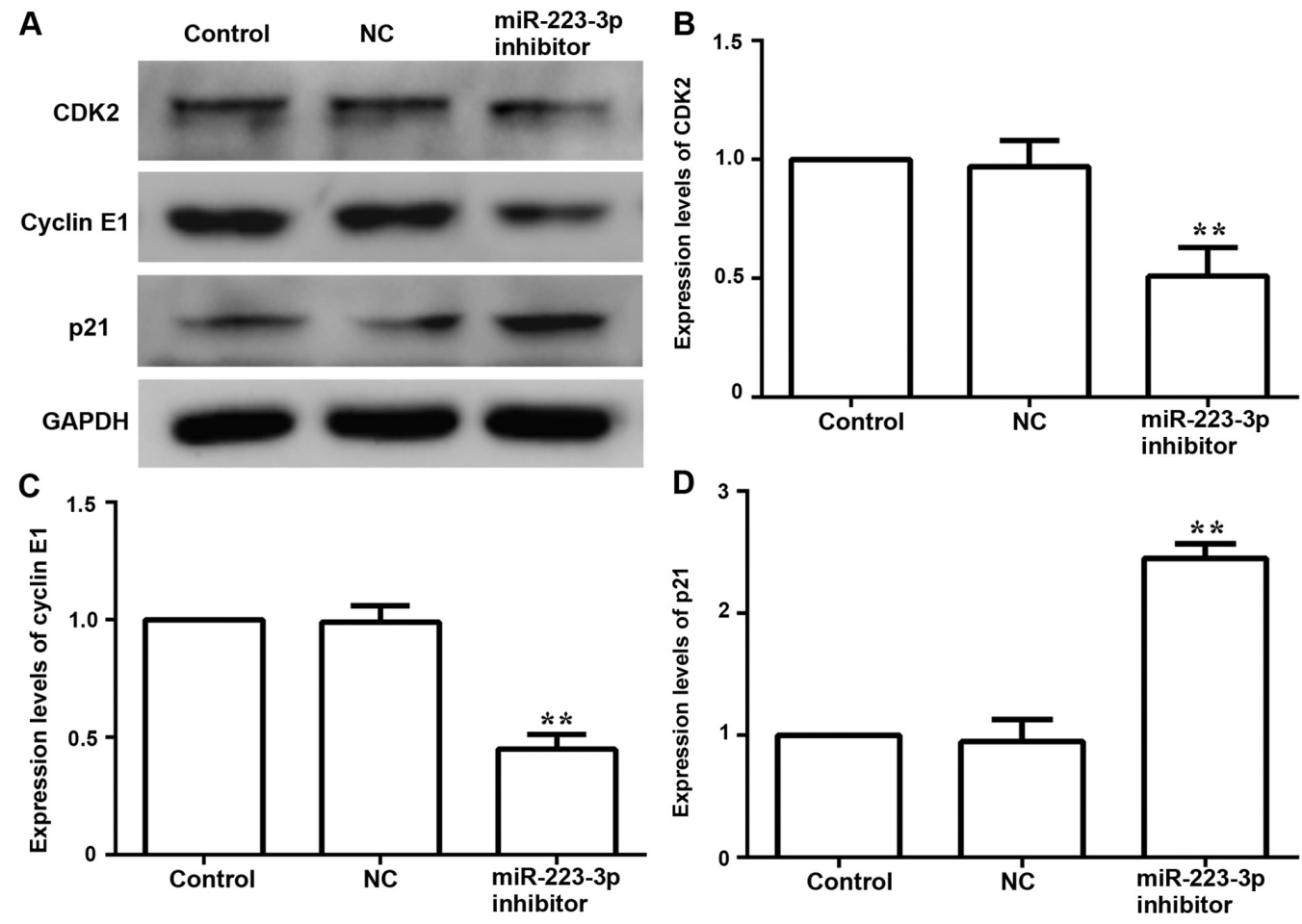

Figure 3. Effects of miR-223-3p inhibition on cell cycle-related protein expression in MCF-7 cells. (A) Representative western blotting results and subsequent densitometric analysis of the protein expression levels of (B) CDK2, (C) cyclin E1 and (D) p21. Data are presented as the mean \pm SD of three experiments. *** $<0.01$ vs. Control. miR, microRNA; NC, negative control; siRNA, small interfering RNA.

\section{Discussion}

The role of miR-223-3p in tumor growth has recently been described. For example, miR-223-3p overexpression promotes the proliferation, migration and invasion of ovarian cancer cells (27). Conversely, ectopic expression of miR-223-3p could inhibit the invasion, migration, growth and proliferation of breast cancer cells (28). It has also been shown that the MMP protein family, including MMP2, MMP9 and MMP14, is involved in cell development and migration (29). In the present study, the expression levels of miR-223-3p were compared between normal and breast cancer cells. It was observed that the transcription levels of miR-223-3p were markedly increased in breast cancer cells. It was also found that following transfection of miR-223-3p inhibitor into cells, miR-223-3p expression could be significantly inhibited. Furthermore, in association with inhibition of miR-223-3p, the activity of tumor cells was markedly decreased, and the apoptosis rate was increased. Thus, the present study demonstrated that miR-223-3p may serve an important role in the proliferation, metastasis and invasion of breast cancer cells.

CDK and the regulatory subunit cyclin can directly affect the progression of the cell cycle (30). The present study demonstrated that miR-223-3p inhibitor markedly inhibited the proliferation of MCF-7 cells by downregulating the expression levels of CDK2 and Cyclin E1, and upregulating p21 expression. In addition, miR-223-3p inhibition significantly decreased the migration and invasion of MCF-7 cells, and the results demonstrated that the expression levels of MMP family proteins were inhibited. These characteristics may contribute to the understanding of miR-223-3p expression and the invasive biological behavior of breast cancer cells.
EMT serves a key role in both normal cell development and tumor development (31). EMT is the process of epithelial cells losing epithelial proteins, including E-cadherin, which is responsible for tight junctions (32), and members of the miR-200 family, which help maintain an epithelial phenotype (33). The cell moves towards a more mesenchymal phenotype as it gains mesenchymal markers, such as $\mathrm{N}$-cadherin, which provides the cells with migratory potential, and vimentin and fibronectin, which are proteins excreted to help form the extracellular matrix (34). However, when proteins involved in EMT are dysregulated, epithelial cell polarity is lost, which increases the invasive ability of tumor cells and accelerates the occurrence and development of tumors (35). EMT serves an important role in cancer progression and can stimulate cells to migrate and invade, increasing their metastatic potential $(36,37)$. Therefore, it was hypothesized that miR-223-3p may affect EMT in breast cancer cells. The present results demonstrated that miR-223-3p inhibition significantly increased the expression levels of the epithelial marker E-cadherin and significantly decreased the expression levels of mesenchymal markers, such as N-cadherin and vimentin, suggesting that miR-223-3p may affect the migration and invasion of MCF-7 cells and aggravate the occurrence of breast cancer. However, compared with control cells, inhibition of miR-223-3p could increase the expression levels of E-cadherin and decrease the expression levels of $\mathrm{N}$-cadherin and vimentin, suggesting that decreasing miR-223-3p expression could inhibit the progression of EMT, and thus inhibit the invasiveness of tumors, which was consistent with the effect of inhibiting the transcription of miR-223-3p on the proliferative, migratory and invasive abilities of MCF-7 cells. However, the mechanism through which miR-223-3p induces EMT requires further clarification. 


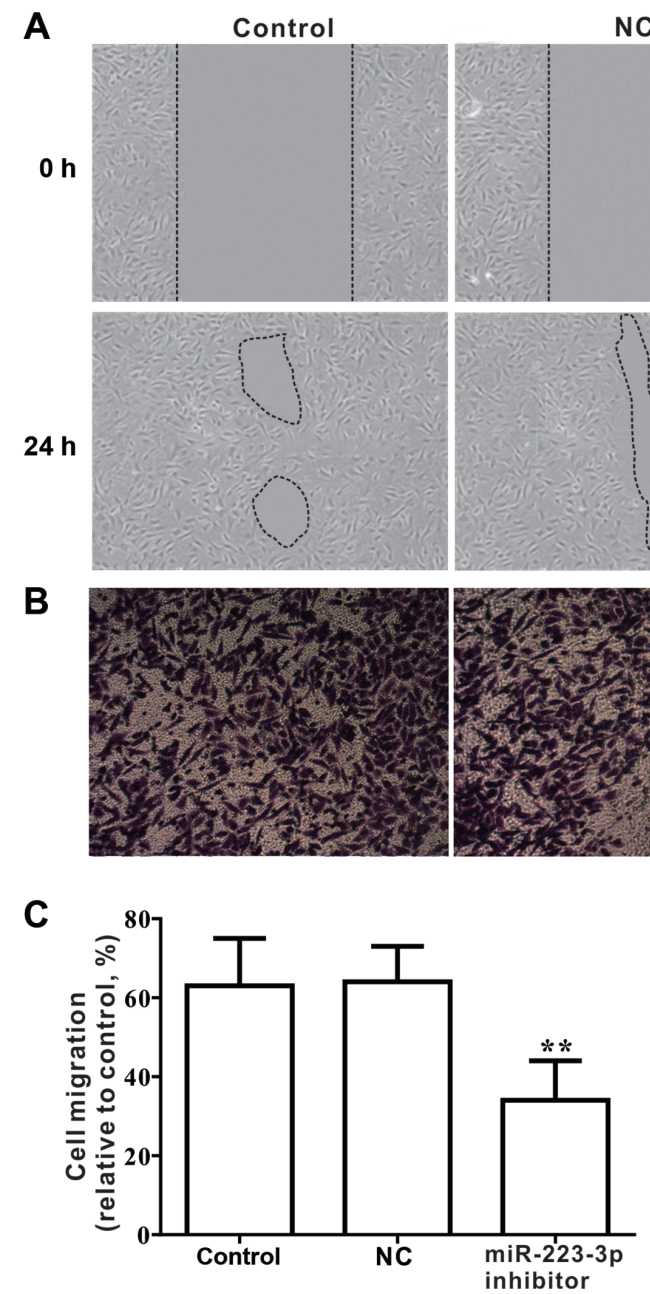

NC miR-223-3p inhibitor

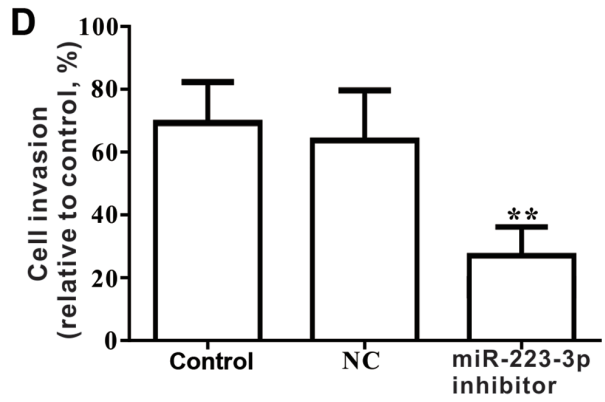

Figure 4. Effects of miR-223-3p inhibition on the migration and invasion of MCF-7 cells. (A) Wound healing assay was performed to detect cell migratory ability; magnification, x100. (B) Transwell assay was performed to detect cell invasive ability; magnification, x200. (C) The 24-h migration rate of MCF-7 cells was calculated after the three indicated treatments. (D) Cell invasion activity was determined using Matrigel invasion assays 24-h after transfection. Data are presented as the mean \pm SD of three experiments. ${ }^{* *} \mathrm{P}<0.01$ vs. Control. miR, microRNA; NC, negative control; siRNA, small interfering RNA.
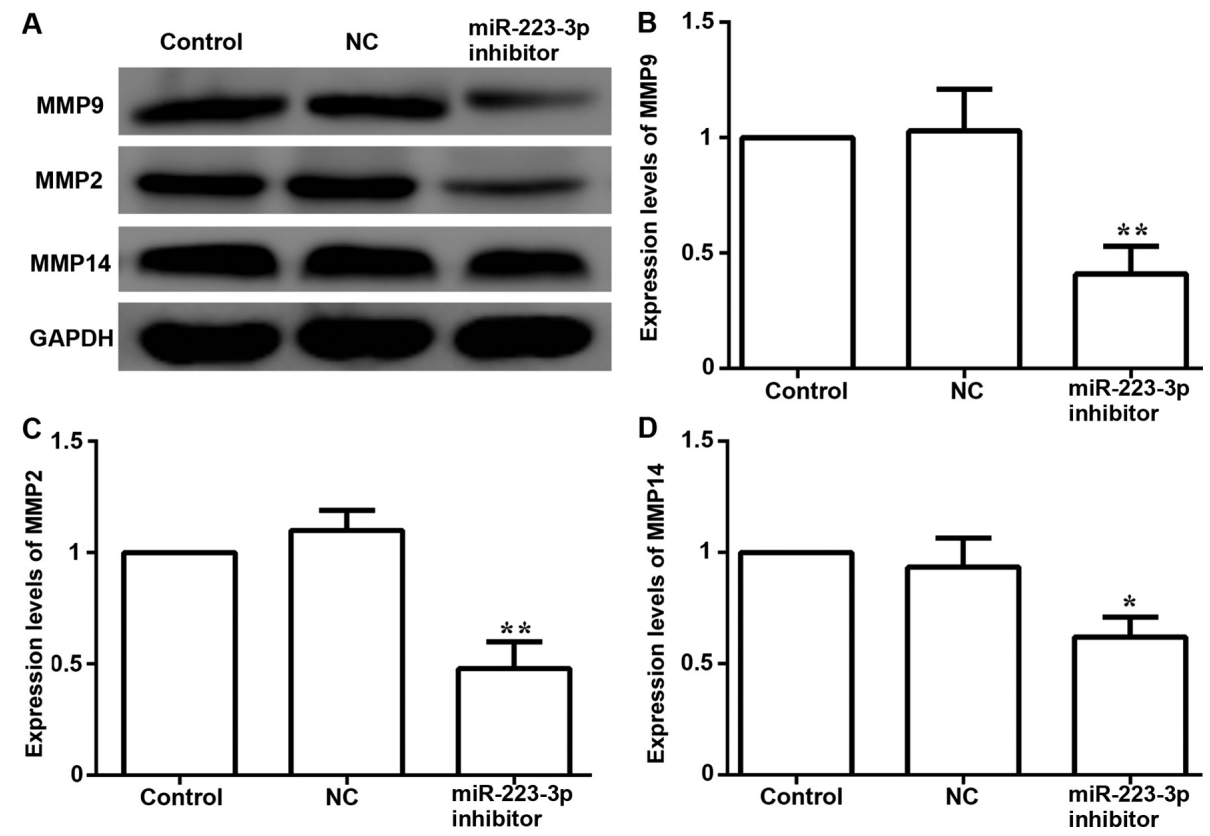

Figure 5. Effects of miR-223-3p inhibition on metastasis-related proteins in MCF-7 cells. (A) Representative western blotting results and subsequent densitometric analysis of the protein expression levels (B) MMP9, (C) MMP2 and (D) MMP14. Data are presented as the mean \pm SD of three experiments. ${ }^{*} \mathrm{P}<0.05$, ${ }^{* *} \mathrm{P}<0.01$ vs. Control. miR, microRNA; NC, negative control; siRNA, small interfering RNA. 

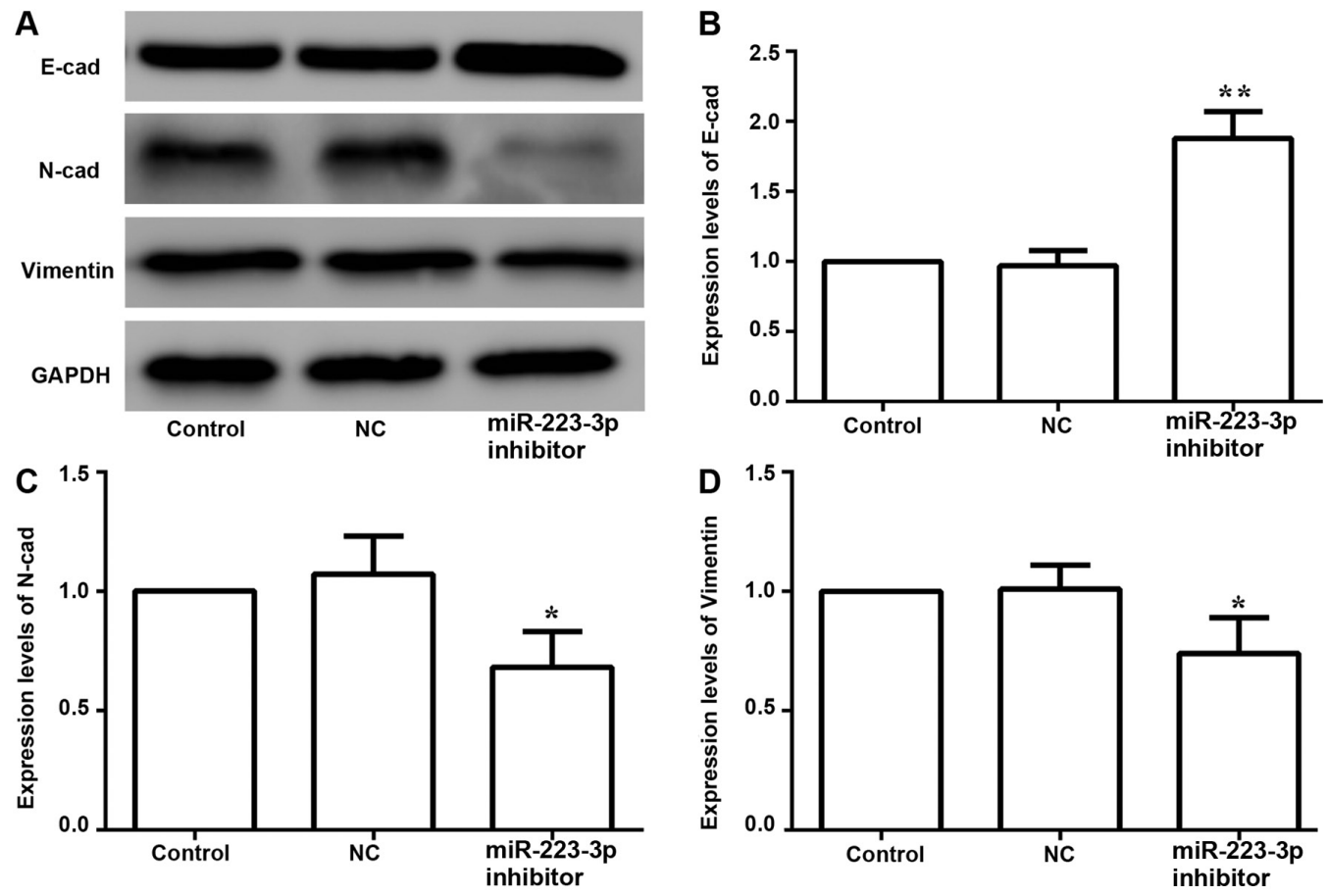

Figure 6. Effects of miR-223-3p inhibition on EMT of breast cancer cells. (A) Representative western blotting results and subsequent densitometric analysis of the expression levels of the EMT-associated proteins (B) E-cad, (C) N-cad and (D) vimentin. Data are presented as the mean \pm SD of three experiments. "P $<0.05,{ }^{* *} \mathrm{P}<0.01$ vs. Control. Cad, cadherin; miR, microRNA; NC, negative control; siRNA, small interfering RNA.
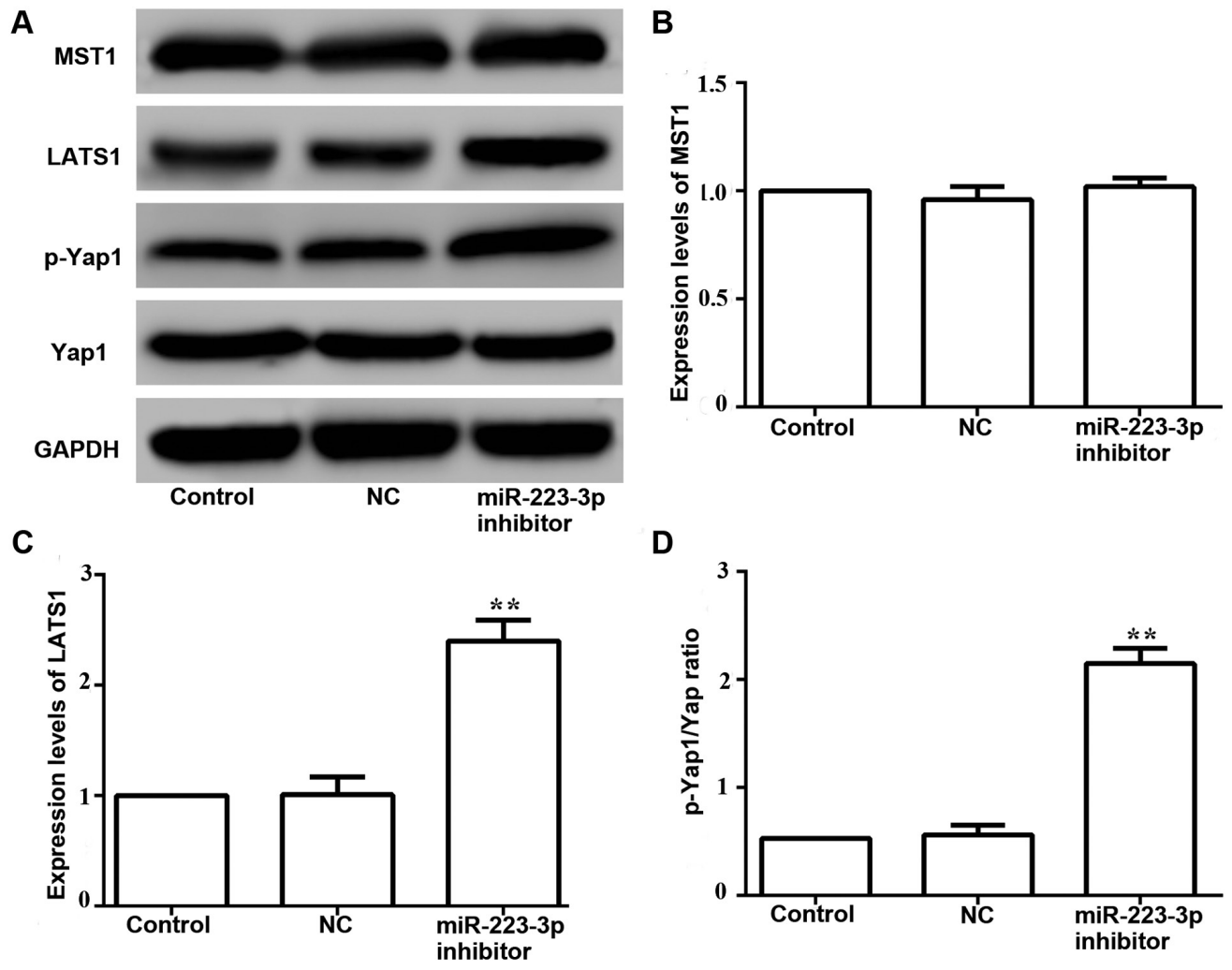

Figure 7. Effects of miR-223-3p inhibition on the Hippo/Yap pathway in breast cancer cells. (A) Representative western blotting results and subsequent densitometric analysis of the expression levels of proteins associated with the Hippo/Yap signaling. Optical density analysis of (B) MST1, (C) LATS1 and (D) YAP protein expression. ${ }^{* *} \mathrm{P}<0.01$ vs. Control. LATS1, large tumor suppressor kinase 1; miR, microRNA; MST1, macrophage stimulating 1; NC, negative control; siRNA, small interfering RNA.

Studies in Drosophila and vertebrates have revealed that the Hippo signaling pathway has important regulatory effects on organ growth (38). As research progresses, increasing evidence suggests that the Hippo signaling pathway is dysregulated in 
the development of human tumors (21). Yap functions as a key downstream effector of the Hippo signaling pathway, mainly through phosphorylation, to inhibit tumor progression (39). Previous studies have reported that the abnormal activation of the Hippo signaling pathway is associated with tumor progression, and abnormal Yap expression is associated with the occurrence of various tumors (38), such as liver cancer (40), breast cancer (40) and pancreatic cancer (41). Studies have also revealed that abnormal activation of Yap serves a key regulatory role in the process of tumorigenesis and development $(39,42,43)$. The present results demonstrated that miR-223-3p inhibition increased the phosphorylation of Yap.

MST1,LATS1 and LATS2 are key kinases in the core kinase cassette of the mammalian Hippo signaling pathway $(44,45)$. Once the Hippo signaling pathway is activated, the MST kinase phosphorylates LATS and the transcriptional co-activator Yap, thereby inactivating Yap (46). Overexpression of Yap can reverse the inhibition of the Hippo signaling pathway by blocking the activity of LATS (47). The present study demonstrated that miR-223-3p inhibition increased the phosphorylation of Yap and upregulated the expression level of the Hippo signaling pathway kinase LATS1. Abnormal Yap overexpression is associated with basic cellular processes, such as cell proliferation, migration, invasion and EMT (48). Therefore, inhibition of miR-223-3p expression may regulate Yap expression via the Hippo signaling pathway, thus inhibiting the proliferation, migration, invasion and EMT of breast cancer cells.

It has been reported that miR-223-3p has the potential to promote the proliferation and invasion of cancer cells (6-11), and the proliferation and invasion of breast cancer cells are significantly enhanced after miR-223-3p transfection into breast cancer cells $(12,49,50)$. The present report, as an initial study of miR-223-3p in breast cancer, aimed to preliminarily investigate whether this miRNA has a regulatory effect on breast cancer cells and to analyze its pathway of action. There are certain limitations, such as the requirement for in vivo experiments to support the present hypothesis. However, relevant animal experiments to verify the regulatory role of miR-223-3p in tumors have been conducted by our group, but relevant experimental data are being submitted, and thus they are not shown in the current manuscript. Future studies will continue to examine the role of miR-223-3p in the occurrence, metastasis and upstream regulators of breast cancer, and investigate whether it affects other tumor regulatory pathways.

In conclusion, the present study demonstrated that miR-223-3p may regulate breast cancer cell proliferation, migration, invasion and EMT via the Hippo/Yap signaling pathway. This phenomenon may be ameliorated after miR-223-3p inhibition. The present results provide a basis for the study of breast cancer and novel ideas for treatment.

\section{Acknowledgements}

Not applicable.

\section{Funding}

The present study was supported by grants from Jilin Provincial Science and Technology Development Project (grant nos. 20200201577JC and 20180520229JH).

\section{Availability of data and materials}

The datasets used and/or analyzed during the current study are available from the corresponding author on reasonable request.

\section{Authors' contributions}

TD performed gene and protein expression, cell viability, apoptosis, cell migration and cell invasion assays. DW performed E-cadherin, N-cadherin and vimentin protein detection. XW performed Mst1, LATS1, Yap1 and phosphorylated Yap1 protein detection. BL designed and supervised the study, and collaborated to discuss the results. JX and QX analyzed the data. TD wrote and modified the manuscript and summarized the experiment based on the data. All authors read and approved the final manuscript.

\section{Ethics approval and consent to participate}

Not applicable.

\section{Patient consent for publication}

Not applicable.

\section{Competing interests}

The authors declare that they have no competing interests.

\section{References}

1. Bray F, Ferlay J, Soerjomataram I, Siegel RL, Torre LA and Jemal A: Global cancer statistics 2018: GLOBOCAN estimates of incidence and mortality worldwide for 36 cancers in 185 countries. CA Cancer J Clin 68: 394-424, 2018.

2. Chen W, Sun K, Zheng R, Zeng H, Zhang S, Xia C, Yang Z, Li H, Zou X and He J: Cancer incidence and mortality in China, 2014. Chin J Cancer Res 30: 1-12, 2018.

3. Rong C, Meinert ÉFRC and Hess J: Estrogen receptor signaling in radiotherapy: From molecular mechanisms to clinical studies. Int J Mol Sci 19: 713, 2018.

4. Al-Mahmood S, Sapiezynski J, Garbuzenko OB and Minko T: Metastatic and triple-negative breast cancer: Challenges and treatment options. Drug Deliv Transl Res 8: 1483-1507, 2018.

5. Jang HJ, Lee HS, Burt BM, Lee GK, Yoon KA, Park YY, Sohn BH, Kim SB, Kim MS, Lee JM, et al: Integrated genomic analysis of recurrence-associated small non-coding RNAs in oesophageal cancer. Gut 66: 215-225, 2017.

6. Mavrakis KJ, Van Der Meulen J, Wolfe AL, Liu X, Mets E, Taghon T, Khan AA, Setty M, Rondou P, Vandenberghe P, et al: A cooperative microRNA-tumor suppressor gene network in acute T-cell lymphoblastic leukemia (T-ALL). Nat Genet 43: 673-678, 2011.

7. Li J, Guo Y, Liang X, Sun M, Wang G, De W and Wu W: MicroRNA-223 functions as an oncogene in human gastric cancer by targeting $\mathrm{FBXW} 7 / \mathrm{hCdc} 4$. J Cancer Res Clin Oncol 138: 763-774, 2012.

8. Wei Y, Yang J, Yi L, Wang Y, Dong Z, Liu Z, Ou-Yang S, Wu H, Zhong Z, Yin Z, et al: MiR-223-3p targeting SEPT6 promotes the biological behavior of prostate cancer. Sci Rep 4: 7546, 2014.

9. Fazi F, Racanicchi S, Zardo G, Starnes LM, Mancini M, Travaglini L, Diverio D, Ammatuna E, Cimino G, Lo-Coco F, et al: Epigenetic silencing of the myelopoiesis regulator microRNA-223 by the AML1/ETO oncoprotein. Cancer Cell 12: 457-466, 2007.

10. Tang Y, Wang Y, Chen Q, Qiu N, Zhao Y and You X: MiR-223 inhibited cell metastasis of human cervical cancer by modulating epithelial-mesenchymal transition. Int J Clin Exp Pathol 8: 11224-11229, 2015. 
11. Zhao FY, Han J, Chen XW, Wang J, Wang XD, Sun JG and Chen ZT: miR-223 enhances the sensitivity of non-small cell lung cancer cells to erlotinib by targeting the insulin-like growth factor-1 receptor. Int J Mol Med 38: 183-191, 2016.

12. Yoshikawa M,Iinuma H,Umemoto Y, Yanagisawa T,Matsumoto A and Jinno H: Exosome-encapsulated microRNA-223-3p as a minimally invasive biomarker for the early detection of invasive breast cancer. Oncol Lett 15: 9584-9592, 2018.

13. Nieto MA, Huang RYJ, Jackson RA and Thiery JP: EMT: 2016 Cell 166: 21-45, 2106 .

14. Wang Y and Zhou BP: Epithelial-mesenchymal transition-a hallmark of breast cancer metastasis. Cancer Hallm 1: 38-49, 2013

15. Ye X, Tam WL, Shibue T, Kaygusuz Y, Reinhardt F, Ng Eaton E and Weinberg RA: Distinct EMT programs control normal mammary stem cells and tumour-initiating cells. Nature 525: 256-260, 2015

16. Krebs AM, Mitschke J, Lasierra Losada M, Schmalhofer O, Boerries M, Busch H, Boettcher M, Mougiakakos D, Reichardt W, Bronsert P, et al: The EMT-activator Zeb1 is a key factor for cel plasticity and promotes metastasis in pancreatic cancer. Nat Cell Biol 19: 518-529, 2017.

17. Mani SA, Guo W, Liao MJ, Eaton EN, Ayyanan A, Zhou AY, Brooks M, Reinhard F, Zhang CC, Shipitsin M, et al: The epithelial-mesenchymal transition generates cells with properties of stem cells. Cell 133: 704-715, 2008 .

18. Singh A and Settleman J: EMT, cancer stem cells and drug resistance: An emerging axis of evil in the war on cancer. Oncogene 29: 4741-4751, 2010.

19. Kar R, Jha NK, Jha SK, Sharma A, Dholpuria S, Asthana N, Chaurasiya K, Singh VK, Burgee S and Nand P: A 'NOTCH' Deeper into the epithelial-to-mesenchymal transition (EMT) program in breast cancer. Genes 10: 961, 2019.

20. Zheng Y and Pan D: The hippo signaling pathway in development and disease. Dev Cell 50: 264-282, 2019.

21. Pan D: The hippo signaling pathway in development and cancer. Dev Cell 19: 491-505, 2010

22. Zhao B, Lei QY and Guan KL: The Hippo-YAP pathway: New connections between regulation of organ size and cancer. Curr Opin Cell Biol 20: 638-646, 2008.

23. Moleirinho S, Chang N, Sims AH, Tilston-Lünel AM, Angus L, Steele A, Boswell V, Barnett SC, Ormandy C, Faratian D, et al: KIBRA exhibits MST-independent functional regulation of the Hippo signaling pathway in mammals. Oncogene 32: 1821-1830, 2013.

24. Liu LL, Zhao H, Ma TF, Ge F, Chen CS and Zhang YP: Identification of valid reference genes for the normalization of RT-qPCR expression studies in human breast cancer cell lines treated with and without transient transfection. PLoS One 10: e0117058, 2015.

25. Livak KJ and Schmittgen TD: Analysis of relative gene expression data using real-time quantitative PCR and the 2(-Delta Delta C(T)) method. Methods 25: 402-408, 2001.

26. Azimi A,Majidinia M, Shafiei-Irannejad V, Jahanban-Esfahlan R, Ahmadi Y, Karimian A, Mir SM, Karami H and Yousefi B: Suppression of p53R2 gene expression with specific siRNA sensitizes HepG2 cells to doxorubicin. Gene 642: 249-255, 2017

27. Fang G, Liu J, Wang Q, Huang X, Yang R, Pang Y and Yang M: MicroRNA-223-3p regulates ovarian cancer cell proliferation and invasion by targeting SOX11 expression. Int J Mol Sci 18: 1208, 2017.

28. Ji Q, Xu X, Song Q, Xu Y, Tai Y, Goodman SB, Bi W, Xu M, Jiao S, Maloney WJ and Wang Y: miR-223-3p inhibits human osteosarcoma metastasis and progression by directly targeting CDH6. Mol Ther 26: 1299-1312, 2018.

29. Jabłońska-Trypuć A, Matejczyk M and Rosochacki S: Matrix metalloproteinases (MMPs), the main extracellular matrix (ECM) enzymes in collagen degradation, as a target for anticancer drugs. J Enzyme Inhib Med Chem 31: 177-183, 2016.

30. Song X, Zhu M, Zhang F, Zhang F, Zhang Y, Hu Y, Jiang L, Hao Y, Chen S, Zhu Q, et al: ZFX promotes proliferation and metastasis of pancreatic cancer cells via the MAPK pathway. Cell Physiol Biochem 48: 274-284, 2018.

31. Iwatsuki M, Mimori K, Yokobori T, Ishi H, Beppu T, Nakamori S, Baba $\mathrm{H}$ and Mori M: Epithelial-mesenchymal transition in cancer development and its clinical significance. Cancer Sci 101: 293-299, 2010.
32. Yang ZC, Yi MJ, Ran N, Wang C, Fu P, Feng XY, Xu L and $\mathrm{Qu} \mathrm{ZH}$ : Transforming growth factor- $\beta 1$ induces bronchial epithelial cells to mesenchymal transition by activating the Snail pathway and promotes airway remodeling in asthma. Mol Med Rep 8: 1663-1668, 2013

33. Korpal M, Lee ES, Hu G and Kang Y: The miR-200 family inhibits epithelial-mesenchymal transition and cancer cell migration by direct targeting of E-cadherin transcriptional repressors ZEB1 and ZEB2. J Biol Chem 283: 14910-14914, 2008.

34. Rout-Pitt N, Farrow N, Parsons D and Donnelley M: Epithelial mesenchymal transition (EMT): A universal process in lung diseases with implications for cystic fibrosis pathophysiology. Respir Res 19: 136, 2018.

35. Royer C and Lu X: Epithelial cell polarity: A major gatekeeper against cancer? Cell Death Differ 18: 1470-1477, 2011

36. Mathias RA, Gopal SK and Simpson RJ: Contribution of cells undergoing epithelial-mesenchymal transition to the tumor microenvironment. J Proteomics 78: 545-557, 2012.

37. Lv B, Yang X, Lv S, Wang L, Fan K, Shi R, Wang F, Song H, Ma X, Tan X, et al: Retraction note to: CXCR4 signaling induced epithelial-mesenchymal transition by PI3K/AKT and ERK pathways in glioblastoma. Mol Neurobiol 54: 2380-2380, 2017.

38. Badouel C, Garg A and McNeill H: Herding Hippos: Regulating growth in flies and man. Curr Opin Cell Biol 21: 837-843, 2009.

39. Ehmer U and Sage J: Control of proliferation and cancer growth by the Hippo signaling pathway. Mol Cancer Res 14: 127-140, 2016.

40. Wang J, Ma L, Weng W, Qiao Y, Zhang Y, He J, Wang H, Xiao W, Li L, Chu Q, et al: Mutual interaction between YAP and CREB promotes tumorigenesis in liver cancer. Hepatology 58: 1011-1020, 2013

41. Jiang Z, Zhou C, Cheng L, Yan B, Chen K, Chen X, Zong L, Lei J, Duan W, Xu Q, et al: Inhibiting YAP expression suppresses pancreatic cancer progression by disrupting tumor-stromal interactions. J Exp Clin Cancer Res 37: 69, 2018.

42. Liu H, Du S, Lei T, Wang H, He X, Tong R and Wang Y: Multifaceted regulation and functions of YAP/TAZ in tumors (Review). Oncol Rep 40: 16-28, 2018.

43. Zanconato F, Cordenonsi $\mathrm{M}$ and Piccolo S: YAP and TAZ: A signalling hub of the tumour microenvironment. Nat Rev Cancer 19: 454-464, 2019.

44. Ma Y, Yang Y, Wang F, Wei Q and Qin H: Hippo-YAP signaling pathway: A new paradigm for cancer therapy. Int J Cancer 137: 2275-2286, 2015

45. Hergovich A: The roles of NDR protein kinases in Hippo signalling. Genes 7: 21, 2016.

46. Zhao B, Lei Q and Guan KL: Mst out and HCC in. Cancer Cell 16: 363-364, 2009.

47. Basu-Roy U, Bayin NS, Rattanakorn K, Han E, Placantonakis DG, Mansukhani A and Basilico C: Sox2 antagonizes the Hippo pathway to maintain stemness in cancer cells. Nat Commun 6: 6411, 2015.

48. Zhang H, Liu CY, Zha ZY, Zhao B, Yao J, Zhao S, Xiong Y, Lei QY and Guan KL: TEAD transcription factors mediate the function of TAZ in cell growth and epithelial-mesenchymal transition. J Biol Chem 284: 13355-13362, 2009.

49. Yang M, Chen J, Su F, Yu B, Su F, Lin L, Liu Y, Huang JD and Song E: Microvesicles secreted by macrophages shuttle invasion-potentiating microRNAs into breast cancer cells. Mol Cancer 10: 117, 2011

50. Cao L, Zhang X, Cao F, Wang Y, Shen Y, Yang C, Uzan G, Peng $B$ and Zhang D: Inhibiting inducible miR-223 further reduces viable cells in human cancer cell lines MCF-7 and PC3 treated by celastrol. BMC Cancer 15: 873, 2015.

This work is licensed under a Creative Commons Attribution-NonCommercial-NoDerivatives 4.0 International (CC BY-NC-ND 4.0) License. 